\title{
Editorial
}

\section{Well-Posed and Ill-Posed Boundary Value Problems for PDE 2013}

\section{Allaberen Ashyralyev, ${ }^{1,2}$ Sergey Piskarev, ${ }^{3}$ Valery Covachev, ${ }^{4}$ Ravshan Ashurov, ${ }^{5}$ Hasan Ali Yurtsever, ${ }^{6}$ and Abdullah Said Erdogan ${ }^{1,7}$}

${ }^{1}$ Department of Mathematics, Fatih University, Buyukcekmece, 34500 Istanbul, Turkey

${ }^{2}$ ITTU, 74400 Ashgabat, Turkmenistan

${ }^{3}$ Science Research Computer Center of Moscow State University, Vorobjevy Gory, Moscow 119899, Russia

${ }^{4}$ Department of Mathematics and Statistics, College of Science, Sultan Qaboos University, 123 Muscat, Oman

${ }^{5}$ The Institute of Mathematics, National University of Uzbekistan, 700095 Tashkent, Uzbekistan

${ }^{6}$ American Islamic College, Chicago, IL 60613, USA

${ }^{7}$ Department of Mathematics, University of Central Florida, Orlando, FL 32816, USA

Correspondence should be addressed to Allaberen Ashyralyev; aashyr@fatih.edu.tr

Received 28 November 2013; Accepted 28 November 2013

Copyright (C) 2013 Allaberen Ashyralyev et al. This is an open access article distributed under the Creative Commons Attribution License, which permits unrestricted use, distribution, and reproduction in any medium, provided the original work is properly cited.

The studies of well-posed and ill-posed boundary value problems for partial differential equations are driven not only by a theoretical interest but also by the fact of several phenomena in engineering and various fields of physics and applied sciences. The present special issue is devoted to the publication of high-quality research papers in the fields of the study of analytic and numerical methods for solutions of well-posed and ill-posed boundary value problems for partial differential equations.

The issue covers a wide variety of problems for different classes of partial differential equations. The topics discussed in the contributed papers are traditional for qualitative theory of differential equations. The issue contains papers on the existence, uniqueness, and asymptotic behavior of a classical solution to the initial and Neumann boundary value problem for a class of nonlinear parabolic equations of Monge-Ampere type and on the blow-up phenomena for a modified twocomponent Dullin-Gottwald-Holm shallow water system. Some new blow-up criteria of strong solutions involving the density and suitable integral form of the momentum are established. Furthermore, an analytical solution for effect of magnetic field and initial stress on an infinite generalized thermoelastic rotating nonhomogeneous diffusion in a medium subjected to certain boundary conditions is studied.
The chemical potential is also assumed to be a known function of time at the boundary of the cavity. The analytical expressions for the displacements, stresses, temperature, concentration, and chemical potential are obtained. Comparison was made with the results obtained in the presence and absence of diffusion. The results indicate that the effects of nonhomogeneity, rotation, magnetic field, relaxation time and diffusion are very pronounced.

A number of papers are concerned with well-posedness of difference schemes for approximate solutions of partial differential equations. Interesting stability and coercive stability estimates are established for solutions of the first and second order of accuracy difference schemes for the inverse problem of the multidimensional elliptic equation with overdetermination. The algorithm for approximate solution is tested in a two-dimensional inverse elliptic problem. Moreover, stability estimates are established for the solution of the first order of accuracy difference scheme for the approximate solution of the determination of a control parameter problem for Schrodinger equations. One paper collected in this special issue addresses construction and investigation of a third order of accuracy absolutely stable difference schemes for the nonlocal boundary value hyperbolic problem. The stability estimates for the solution of this difference scheme are 
established. Two authors deal with analysis of the block-grid method for the solution of Laplace's equation on polygons with a slit. The error estimates obtained for solving Laplace's boundary value problem on polygons by the block-grid method contain constants that are difficult to calculate accurately. Therefore, the experimental analysis of the method could be essential. The real characteristics of the block-grid method for solving Laplace's equation on polygons with a slit are analysed by experimental investigations. The numerical results obtained show that the order of convergence of the approximate solution is the same as in the case of a smooth solution. To illustrate the singular behaviour around the singular point, the shape of the highly accurate approximate solution and the figures of its partial derivatives up to second order are given in the singular part of the domain. Finally a highly accurate formula is given to calculate the stress intensity factor, which is an important quantity in fracture mechanics.

The issue contains papers on the spectrum of differential operators and its applications. The nature of the spectrum of the periodic problem for the heat equation with a lowerorder term and with a deviating argument is investigated. A significant influence of the lower-order term on the correct solvability of this problem is obtained. A criterion for the strong solvability of the above-mentioned problem is obtained. One paper deals with a Dirac system with transmission condition and eigenparameter in boundary condition. Some spectral properties of the problem are studied. Finally, spectral properties of Sturm-Liouville type problems with interior singularities are investigated. Special solutions of the homogeneous equation are presented.

Finally, the theory of contrasting structures in singularly perturbed boundary problems for nonlinear parabolic partial differential equations is applied to the research of formation of steady state distributions of power within the nonlinear power-society model. The interpretations of the solutions to the equation are presented in terms of applied model. The possibility theorem for the problem of getting the solution having some preassigned properties by means of parametric control is proved.

The volume is a collection of 12 accepted manuscripts by 23 authors. The selection of the papers included in this volume was based on an international peer review procedure. The accepted manuscripts examine wide ranging and cutting edge developments in various areas of well-posed and illposed boundary value problems for partial differential equations. The papers give a taste of current research. We feel the variety of topics will be of interest to both graduate students and researchers.

Further, we are very grateful to all authors for sending their valuable papers for the publication in the present special issue.

Allaberen Ashyralyev Sergey Piskarev Valery Covachev Ravshan Ashurov Hasan Ali Yurtsever Abdullah Said Erdogan 


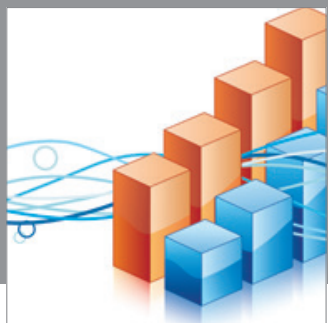

Advances in

Operations Research

mansans

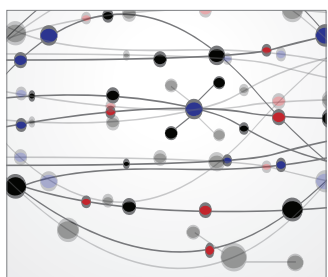

The Scientific World Journal
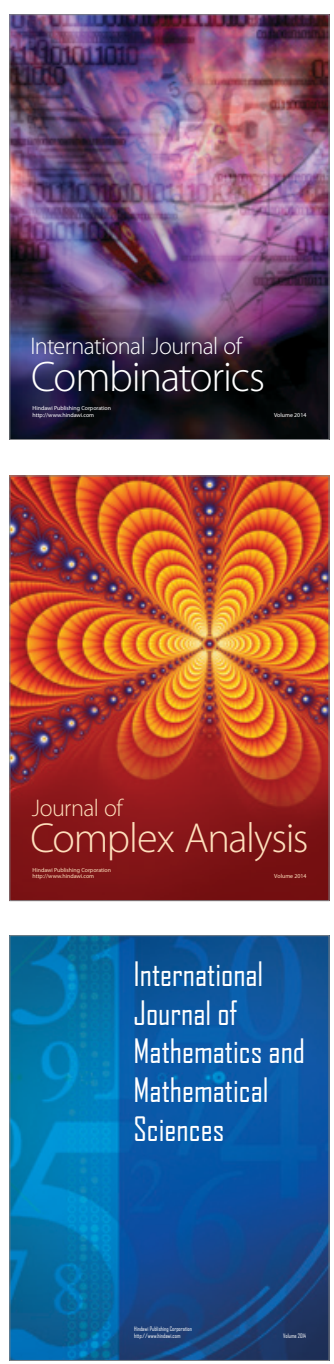
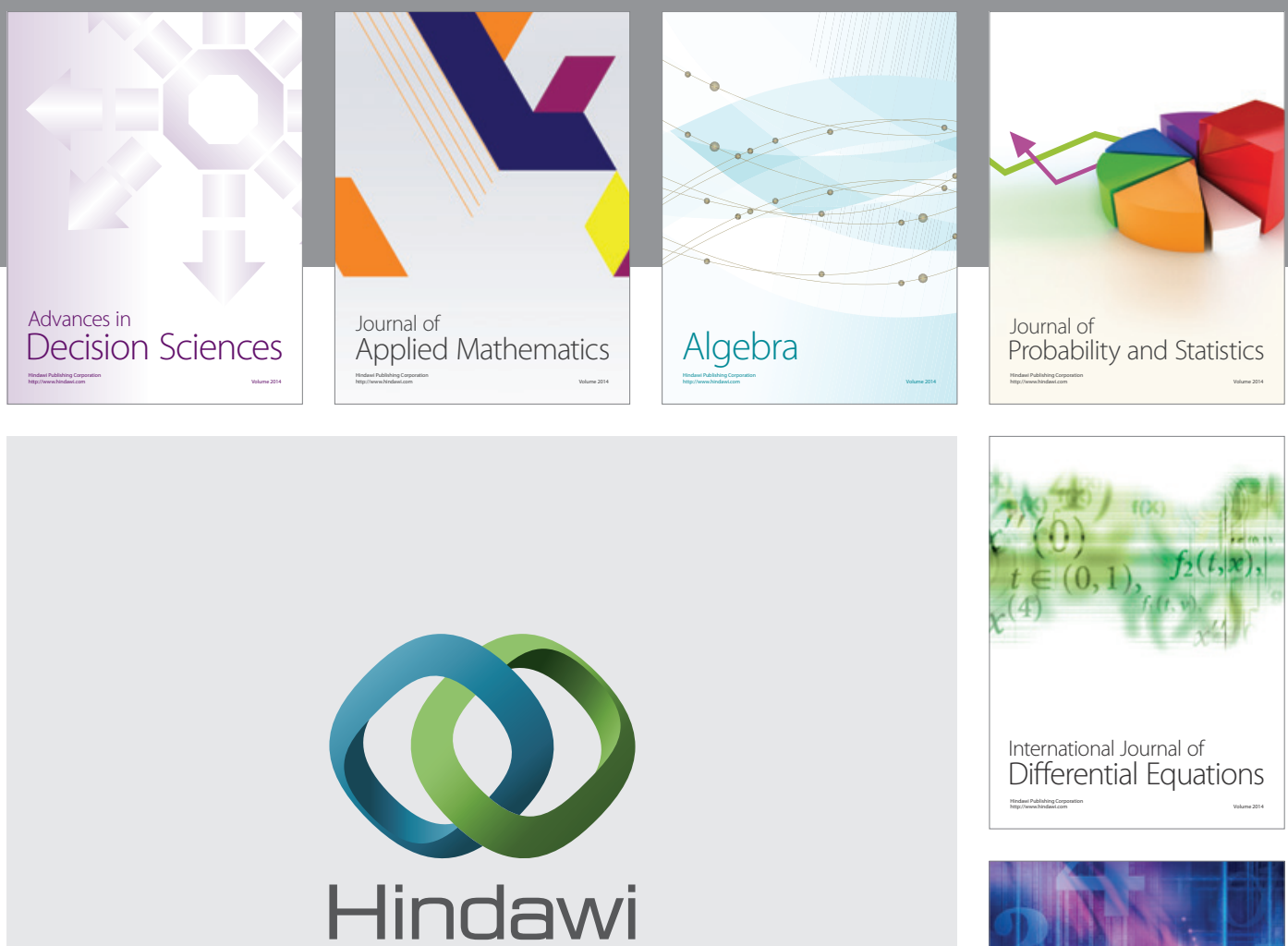

Submit your manuscripts at http://www.hindawi.com
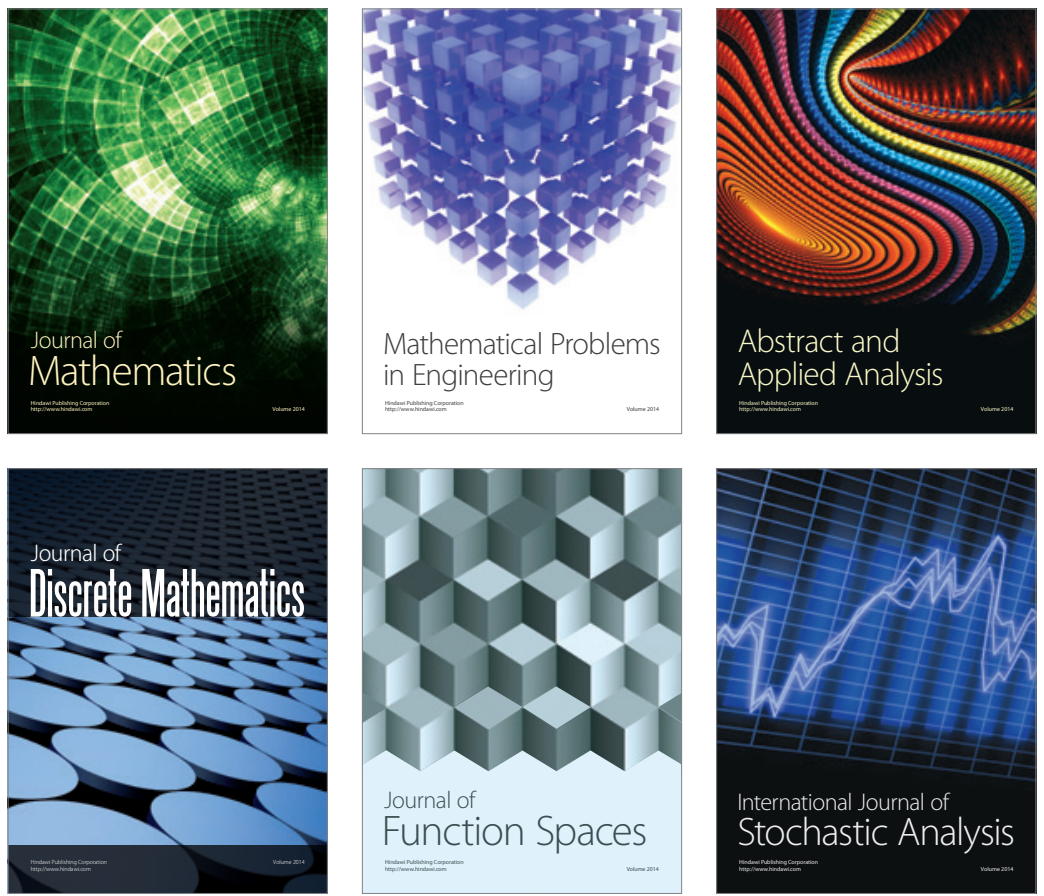

Journal of

Function Spaces

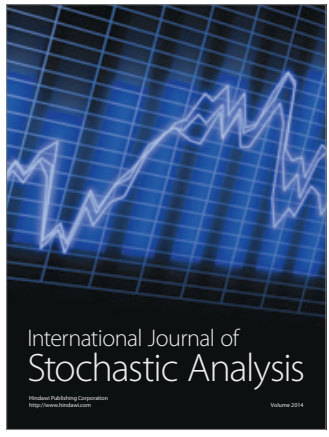

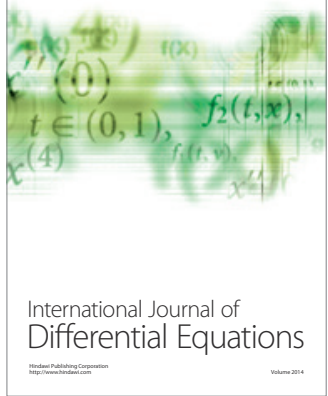
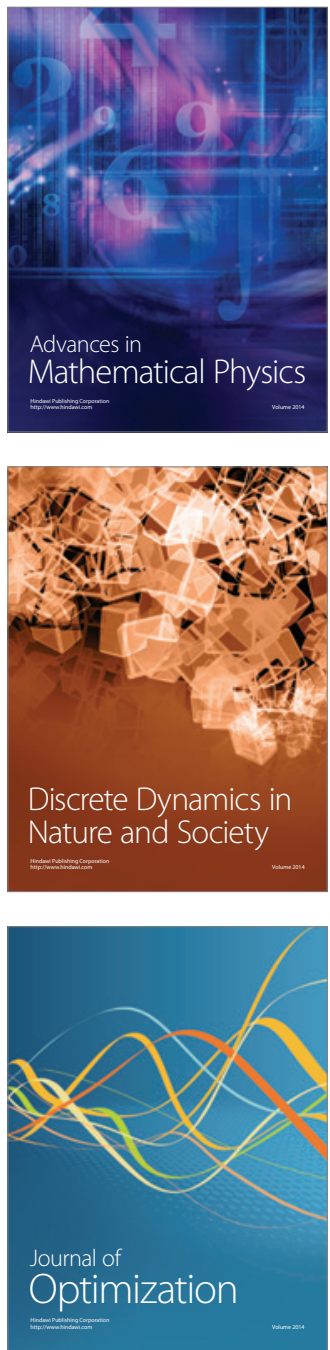